\title{
Simulation Methods of Multivariate Normal Distribution
}

\author{
Fadhil Abdul Abbas Abidy \\ Technical College of Management \\ Al-Furat Al-Awsat Technical University \\ abidy_fadhil@yahoo.com
}

Recived : $8 \backslash 10 \backslash 2017$

Available online : $26 / 1 / 2018$

DOI: $10.29304 / j q c m .2018 .10 .1 .358$

\author{
Ali Hussein Battor Esraa Abdul Reza Baqir \\ Department of Mathematics \\ College of Education for Girls \\ University of Kufa \\ alih.battor@uokufa.edu.iq asraalghanm@gamil.com
}

Revised : //

Accepted : 3\12\2017

\begin{abstract}
In this paper, we are studying three simulation methods to generate observation for multivariate normal distribution, and these methods are: Matlab mvnrnd, decomposition and conditional methods, and we put simulation programs for each method by Matlab 2015a software, and comparison between these methods by depend on many criterions as MSE, AIC, skw, kur. As well as the run speed criterion for each method to get the best method.
\end{abstract}

\section{1- Introduction:}

Wolfgang Bischoff and Werner Fieger generalization the result of the Castillo and Galambos for multivariate random vectors [4], Patrick J., Matias, katarzyna [5], Chun-Chao Wang [6] and others wrote about the multivariate normal distribution and the simulation tests.

Multivariate distributions are studying with several variables (p)that is associated with each relationship and different degrees and thus dependent with a variance and covariance matrix $\Sigma$.[1] If $Y$ has a multivariate normal distribution with mean vector $\mu$ and var-covariance matrix $\sum$, the density function is given by :

$$
g(Y)=\frac{1}{(\sqrt{2 \pi})^{p}|\Sigma|^{\frac{1}{2}}} e^{-(Y-\mu)^{\prime} \Sigma^{-1}(Y-\mu) / 2}
$$

Where $\mu$ :Length-p row vector, $\Sigma: p \times p$ Matrix, $|\Sigma|$ :Matrix determinant, and $p$ is the number of variables. [2]

\section{2- The concept of simulation}

As a result of appearance several problems and statistical theories which are difficult find a logical analysis by mathematical proof, so it has been translated and transformation these theories to real societies, then they have chosen a number of independent random samples, To get the ideal solution for these problems, so practically these samples which are difficult find at the area because they Requires High cost, Time and effort hence some researchers have gone in the beginning of Twentieth century to apply technique the sampling experiment that which is known today simulation .The simulation process is a digital style to complete the experiments on the electronic calculator, which include types of logical and mathematical operations necessary to describe the behavior and structure of complex real system through a given time period. 


\section{3- Comparative criteria:}

1) Mean squared error (MSE)

If $\mathrm{T}$ is (statistic) estimate for the parameter $\theta$ then we called that $E\left[(T-\theta)^{2}\right]$ is

$$
\begin{array}{r}
M S E=E\left[(T-\theta)^{2}\right] \\
=V(T)+[\theta-E(T)]^{2}
\end{array}
$$

Now when the estimate $\mathrm{T}$ be unbiased estimator then $\theta=E(T)$, which mean that

$$
[\theta-E(T)]^{2} \text { is equal to zero and }
$$

$$
M S E=V(T) \text {. }
$$

There is another formula for these estimators specially (for joint estimator) of it as

$$
M S E_{\text {model }}=\operatorname{det} \frac{1}{\operatorname{Rep}} \sum_{i=1}^{\operatorname{Rep}}\left((T-\theta)(T-\theta)^{\prime}\right)
$$

Where Rep: Replication of experiment

2) Akaike information criteria (AIC)

The form of this criteria is either

$$
\begin{gathered}
A I C=-2 \log (M L E)+2 n, \text { or } \\
A I C=N \log (M S E)+2 n
\end{gathered}
$$

Where n: number of fitted parameters,

$\mathrm{N}$ : sample size.

3) Mardia's test statistic for skewness and kurtosis [6] [7]

If $X_{1}, X_{2}, \ldots, X_{n}$ random sample of independent and identical p-variate vectors with unknown mean $\mu$ and unknown covariance matrix $\sum$. Mardia (1970-1974) defined the measure of multivariate skewness and kurtosis as follows:

Where

$$
b_{1, p}=\frac{1}{n^{2}} \sum_{i, j=1}^{n} g_{i j}{ }^{3}
$$

And

$$
g_{i j}=\left(x_{i}-\bar{x}\right)^{\prime} S^{-1}\left(x_{j}-\bar{x}\right)
$$

$b_{2, p}=\frac{1}{n} \sum_{i=1}^{n}\left\{\left(x_{i}-\bar{x}\right)^{\prime} S^{-1}\left(x_{i}-\bar{x}\right)\right\}^{2}$

Under normality of $X_{1}, X_{2}, \ldots, X_{n}$, asymptotically $\mathrm{MVN}, \mathrm{A}=\mathrm{n} b_{1, p} / 6$ has a $X^{2}$ distribution with $\mathrm{f}=\mathrm{p}(\mathrm{p}+1)(\mathrm{p}+2) / 6$ degrees of freedom and the statistic $B=b_{2, p}-p(p+2) / \sqrt{8 p(p+2) / n}$ has asymptotic standard normal distribution . Based on the statistic A and B, as test for multivariate normality jarque and bera (1987) proposed to use the statistic $\mathrm{JB}=\mathrm{A}+\mathrm{B}^{2}$ which has asymptotic chi-square distribution with $f+1$ degrees of freedom. in addition, the distribution is symmetric (null of skewness) around the curve when the value of skewness is zero ( $\mathrm{sk}=0$ ), and the value of kurtosis for the normal distribution in univariate case is 3,while the Mardia's kurtosis is $p(p+2)$ for the multivariate distribution of $\mathrm{p}$ - variables, which is $\mathrm{ku}=$ $2(2+2)=8$ when $(p=2)$.but, the p_JB criterion has belong on the following hypothesis

$\mathrm{H}_{\circ}$ : the data is belong to MVN

If $\mathrm{p}$-value $<0.05$ the hypothesis $\mathrm{H} \circ$ is reject. Either $\mathrm{p}$-value $>0.05$ the hypothesis $\mathrm{H}_{\circ}$ is not reject.

\section{4- Formulation of Simulation Model}

We are choose Matlab 2015a as a program for this study to write a simulation model to generate observation for multivariate normal distribution and selecting default value for the parameters $\mu=\left[\begin{array}{c}2.6 \\ 4\end{array}\right], \Sigma=\left[\begin{array}{cc}1 & -1 \\ -1 & 2\end{array}\right]$ of this distribution, in addition to select sample size $n=15$, 50,100 and 200 respectively and choosing the number of replication as $(\mathrm{R}=10000)$.

\section{5- Simulation method}

\section{1- Matlab mvnrnd}

This method depend on the following formula to get observation for multivariate normal distribution as

$$
y=\text { mvnrnd(mu,sigma_cov, sample size })
$$

Where mu: mean value vector for the distribution sigma_cov : variance and cov_variance for the variable and we wrote a complete program for this method in matlab software.

\section{2- Decomposition method}

To generate observation of multivariate normal distribution by this method must be generate the vector $\underline{Z}$ from the relation $Z \sim N_{p}(0,1)$

and also generate the matrix $\mathrm{T}$ from the form $\operatorname{chol} A$ in matlab program, such that A is var_cov matrix , then get the variable $X$ as

$X=\underline{\mu}+T \underline{Z}$ that submit to this distribution.

\section{3- Conditional method[3]}

The idea of this method is

- Generate $x_{1}$ from the marginal distribution of $X_{1}$.

- Generate $x_{2}$ from the conditional distribution of $X_{2}$, given $X_{1}=x_{1}$.

The suitability of this method for a given bivariate distribution depends on there being an efficient method for generating from the required univariate distributions. 
Let $\left(X_{1}, X_{2}\right)^{\prime}$ denote the bivariate normal vector with covariance matrix $\sum$.define

$$
Y_{2}=\frac{\left(X_{2}-\mu_{2}\right)-\frac{\sigma_{2}}{\sigma_{1}}\left(X_{1}-\mu_{1}\right) \rho}{\sigma_{2}\left(1-\rho^{2}\right)^{1 / 2}}
$$

Then $Y_{1}$ and $Y_{2}$ are two independent standard normal variables, and we can express it by

$$
\begin{gathered}
X_{1}=\sigma_{1} Y_{1}+\mu_{1} \\
X_{2}=\sigma_{2} \rho Y_{1}+\sigma_{2}\left(1-\rho^{2}\right)^{\frac{1}{2}} Y_{1}+\mu_{2}
\end{gathered}
$$

Univariate standard generators are widely available for this purpose.
More generally, let $X \sim N\left(\mu, \sum\right)$, i.e, $\mathrm{X}$ is a p-dimensional multivariate normal random vector with mean vector $\mu$ and covariance matrix $\sum$, let $\mathrm{L}$ be the lower triangular matrix of the cholesky decomposition of $\sum$, i.e a matrix such that $\sum=L^{\prime}$ '. ( routines for computing $L$ are available in many computer software packages.) given $\rho$ independent univariate standard variates, $Y^{\prime}=$ $\left(Y_{1}, \ldots, Y_{\rho}\right)$, transform them

$$
X=L Y+\mu
$$

To achieve $N_{p}(\mu, \Sigma)$ distribution.

\section{5- Result of simulation}

After we show the special methods to generate observation for multivariate normal distribution, we

\begin{tabular}{|c|c|c|c|c|c|c|c|c|c|}
\hline $\begin{array}{c}\text { Sample } \\
\text { size }\end{array}$ & $\widehat{\mu}$ & $\widehat{\Sigma}$ & $\widehat{\boldsymbol{\rho}}$ & MSE & $\begin{array}{l}\text { skw } \\
\text { Sig. of } \\
\text { skw }\end{array}$ & $\begin{array}{c}\text { kur } \\
\text { Sig. of } \\
\text { kur }\end{array}$ & $\left(\mathbf{P} \_J B\right)$ & time & AIC \\
\hline 15 & $\begin{array}{l}{[2.5987} \\
3.9979]\end{array}$ & $\begin{array}{l}{[0.9993} \\
1.9968]\end{array}$ & -0.7350 & 3.2092 & $\begin{array}{l}0.4369 \\
0.8955\end{array}$ & $\begin{array}{l}5.7690 \\
0.1401\end{array}$ & 0.9113 & 1.4508 & 21.4905 \\
\hline 50 & $\begin{array}{l}{[2.5981} \\
4.0010]\end{array}$ & $\begin{array}{l}{[0.9948} \\
2.0012]\end{array}$ & -0.7082 & 0.0848 & $\begin{array}{l}0.2766 \\
0.6799\end{array}$ & $\begin{array}{l}6.0539 \\
0.0427\end{array}$ & 0.6620 & 0.9672 & -119.4022 \\
\hline 100 & $\begin{array}{l}{[2.5986} \\
4.0021]\end{array}$ & $\begin{array}{l}1.0006 \\
2.0010]\end{array}$ & -0.7750 & 0.0101 & $\begin{array}{l}0.2457 \\
0.3933\end{array}$ & $\begin{array}{l}7.6275 \\
0.3207\end{array}$ & 0.5616 & 1.1856 & -455.6912 \\
\hline 200 & $\begin{array}{l}{[2.5992} \\
4.0014]\end{array}$ & $\begin{array}{l}{[0.9999} \\
2.0025]\end{array}$ & -0.7266 & 0.0012 & $\begin{array}{l}0.4234 \\
0.0069\end{array}$ & $\begin{array}{l}9.1161 \\
0.0243\end{array}$ & 0.4155 & 2.3244 & -1334.0 \\
\hline
\end{tabular}
review the results that obtained it from these methods as:

Table (3-1): Simulation result of multivariate normal distribution by mvnrnd method

\begin{tabular}{|c|c|c|c|c|c|c|c|c|c|}
\hline $\begin{array}{c}\text { Sample } \\
\text { size }\end{array}$ & $\widehat{\mu}$ & $\widehat{\Sigma}$ & $\widehat{\rho}$ & MSE & $\begin{array}{c}\text { skw } \\
\text { Sig. of } \\
\text { skw }\end{array}$ & $\begin{array}{c}\text { kur } \\
\text { Sig. of } \\
\text { kur }\end{array}$ & (P_JB) & time & AIC \\
\hline 15 & {$[2.5975$} & {$[0.9971$} & $-\mathbf{0 . 8 3 3 0}$ & $\mathbf{3 . 1 4 4 5}$ & $\mathbf{0 . 7 8 7 5}$ & $\mathbf{5 . 7 2 0 7}$ & $\mathbf{0 . 9 4 8 3}$ & $\mathbf{0 . 9 6 7 2}$ & $\mathbf{2 1 . 1 8 4 8}$ \\
& $\mathbf{4 . 0 0 5 6}]$ & $\mathbf{1 . 9 9 1 7 ]}$ & & & $\mathbf{0 . 7 4 1 5}$ & $\mathbf{0 . 1 3 4 9}$ & & & \\
\hline $\mathbf{5 0}$ & {$[2.5996$} & {$[0.9997$} & $\mathbf{- 0 . 8 0 6 4}$ & $\mathbf{0 . 0 8 0 9}$ & $\mathbf{1 . 2 3 7 7}$ & $\mathbf{8 . 0 7 2 7}$ & $\mathbf{0 . 2 8 1 8}$ & $\mathbf{0 . 9 8 2 8}$ & $\mathbf{- 1 2 1 . 6 9 7 1}$ \\
& $\mathbf{3 . 9 9 9 8}]$ & $\mathbf{1 . 9 9 5 7}]$ & & & $\mathbf{0 . 0 3 5 5}$ & $\mathbf{0 . 4 7 4 4}$ & & & \\
\hline 100 & {$[2.5980$} & {$[0.9999$} & $\mathbf{- 0 . 5 7 3 6}$ & $\mathbf{0 . 0 1 0 1}$ & $\mathbf{0 . 1 3 0 7}$ & $\mathbf{7 . 5 3 1 6}$ & $\mathbf{0 . 6 9 7 5}$ & $\mathbf{1 . 1 0 7 6}$ & $\mathbf{- 4 5 5 . 1 0 3 7}$ \\
& $\mathbf{4 . 0 0 2 9}]$ & $\mathbf{1 . 9 9 7 0 ]}$ & & & $\mathbf{0 . 7 0 3 1}$ & $\mathbf{0 . 2 7 9 1}$ & & & \\
\hline 200 & {$[2.5994$} & {$[1.0003$} & $\mathbf{- 0 . 7 0 1 9}$ & $\mathbf{0 . 0 0 1 3}$ & $\mathbf{0 . 0 2 0 1}$ & $\mathbf{7 . 7 2 6 7}$ & $\mathbf{0 . 9 8 8 4}$ & $\mathbf{1 . 2 3 2 4}$ & $\mathbf{- 1 3 3 2 . 4}$ \\
& $\mathbf{3 . 9 9 9 5}]$ & $\mathbf{2 . 0 0 0 8}]$ & & & $\mathbf{0 . 9 5 4 9}$ & $\mathbf{0 . 3 1 4 5}$ & & & \\
\hline
\end{tabular}

Table (3-2): Simulation result of multivariate normal distribution by decomposition method 
Table (3-3): Simulation result of multivariate normal distribution by conditional method

\begin{tabular}{|c|c|c|c|c|c|c|c|c|c|}
\hline $\begin{array}{l}\text { Sample } \\
\text { size }\end{array}$ & $\widehat{\mu}$ & $\widehat{\Sigma}$ & $\widehat{\rho}$ & MSE & $\begin{array}{c}\text { skw } \\
\text { Sig. of } \\
\text { skw }\end{array}$ & $\begin{array}{l}\text { kur } \\
\text { Sig. of } \\
\text { kur }\end{array}$ & (P_JB) & time & AIC \\
\hline 15 & $\begin{array}{l}{[2.6010} \\
4.0007]\end{array}$ & $\begin{array}{l}{[0.9973} \\
1.9966]\end{array}$ & -0.7484 & 3.2385 & $\begin{array}{l}0.5520 \\
0.8477\end{array}$ & $\begin{array}{l}0.9016 \\
0.1548\end{array}$ & 0.7903 & 0.9204 & 21.6265 \\
\hline 50 & $\begin{array}{l}{[2.6007} \\
3.9996]\end{array}$ & $\begin{array}{l}{[1.0028} \\
1.9972]\end{array}$ & -0.6849 & 0.0842 & $\begin{array}{l}0.2019 \\
0.7939\end{array}$ & $\begin{array}{l}7.2519 \\
0.2542\end{array}$ & 0.7057 & 0.9516 & -119.7166 \\
\hline 100 & $\begin{array}{l}{[2.6014} \\
3.9989]\end{array}$ & $\begin{array}{l}{[0.9997} \\
1.9997]\end{array}$ & -0.7280 & 0.0104 & $\begin{array}{l}0.2071 \\
0.4852\end{array}$ & $\begin{array}{l}8.2721 \\
0.3669\end{array}$ & 0.1768 & 1.0296 & -452.1387 \\
\hline 200 & $\begin{array}{l}{[2.6001} \\
4.0002]\end{array}$ & $\begin{array}{l}{[1.0006} \\
1.9998]\end{array}$ & -0.6959 & 0.0012 & $\begin{array}{l}0.0445 \\
0.8295\end{array}$ & $\begin{array}{l}8.0473 \\
0.4666\end{array}$ & 0.8811 & 1.2480 & -1334.2 \\
\hline
\end{tabular}

Table (3-4): Comparison between the results in table (3-1),(3-2)and (3-3)

\begin{tabular}{|c|c|c|c|c|c|c|c|c|c|c|}
\hline $\begin{array}{c}\text { Sample } \\
\text { size }\end{array}$ & methods & $\widehat{\mu}$ & $\widehat{\Sigma}$ & $\widehat{\rho}$ & MSE & $\begin{array}{c}\text { skw } \\
\text { Sig. of } \\
\text { skw }\end{array}$ & $\begin{array}{c}\text { kur } \\
\text { Sig. of } \\
\text { kur }\end{array}$ & $\left(\mathbf{P} \_\right.$JB $)$ & time & AIC \\
\hline \multirow{3}{*}{15} & $\begin{array}{c}\text { Matlab } \\
\text { (mvnrnd) }\end{array}$ & $\begin{array}{l}{[2.5975} \\
4.0056]\end{array}$ & $\begin{array}{l}{[0.9971} \\
1.9917]\end{array}$ & $-\mathbf{0 . 8 3 3 0}$ & $3.1445 *$ & $\begin{array}{l}0.7875 \\
0.7415\end{array}$ & $\begin{array}{l}\mathbf{5 . 7 2 0 7} \\
\mathbf{0 . 1 3 4 9}\end{array}$ & 0.9483* & 0.9672 & 21.1848* \\
\hline & Decomposition & $\begin{array}{l}{[2.5987} \\
3.9979]\end{array}$ & $\begin{array}{l}{[0.9993} \\
1.9968]\end{array}$ & $\begin{array}{l}- \\
0.7350 *\end{array}$ & 3.2092 & $\begin{array}{l}0.4369 * \\
0.8955\end{array}$ & $\begin{array}{c}5.7690 \\
0.1401\end{array}$ & 0.9113 & 1.4508 & 21.4905 \\
\hline & Conditional & $\begin{array}{l}{[2.6010} \\
4.0007]\end{array}$ & $\begin{array}{l}{[0.9973} \\
1.9966]\end{array}$ & -0.7484 & 3.2385 & $\begin{array}{c}0.5520 \\
0.8477\end{array}$ & $\begin{array}{l}5.9016 * \\
0.1548\end{array}$ & 0.7903 & $0.9204 *$ & 21.6265 \\
\hline \multirow{3}{*}{50} & $\begin{array}{c}\text { Matlab } \\
\text { (mvnrnd) }\end{array}$ & $\begin{array}{l}{[2.5996} \\
3.9998]\end{array}$ & $\begin{array}{l}{[0.9997} \\
1.9957]\end{array}$ & -0.8064 & 0.0809* & $\begin{array}{l}1.2377 \\
0.0355\end{array}$ & $\begin{array}{c}8.0727 * \\
0.4744\end{array}$ & 0.2818 & 0.9828 & $\begin{array}{l}- \\
121.6971 *\end{array}$ \\
\hline & osition & $\begin{array}{l}{[2.5981} \\
4.0010]\end{array}$ & $\begin{array}{l}{[0.9948} \\
2.0012]\end{array}$ & $\begin{array}{l}\overline{0} \\
0.7082 *\end{array}$ & 0.0848 & $\begin{array}{c}0.2766 \\
0.6799\end{array}$ & $\begin{array}{c}6.0539 \\
0.0427\end{array}$ & 0.6620 & 0.9672 & -119.4022 \\
\hline & Conditional & $\begin{array}{l}{[2.6007} \\
3.9996]\end{array}$ & $\begin{array}{l}{[1.0028} \\
1.9972]\end{array}$ & -0.6849 & 842 & $\begin{array}{l}0.2019 * \\
0.7939\end{array}$ & $\begin{array}{c}7.2519 \\
0.2542\end{array}$ & $0.7057 *$ & $0.9516 *$ & -119.7166 \\
\hline \multirow{3}{*}{100} & $\begin{array}{c}\text { Matlab } \\
\text { (mvnrnd) }\end{array}$ & $\begin{array}{l}{[2.5980} \\
4.0029]\end{array}$ & $\begin{array}{l}{[0.9999} \\
1.9970]\end{array}$ & -0.5736 & 0.0101* & $\begin{array}{c}0.1307 * \\
0.7031\end{array}$ & $\begin{array}{l}7.5316 \\
0.2791\end{array}$ & $0.6975 *$ & 1.1076 & -455.1037 \\
\hline & Decomposition & $\begin{array}{l}{[2.5986} \\
4.0021]\end{array}$ & $\begin{array}{l}1.0006 \\
2.0010\end{array}$ & -0.7750 & 0.0102 & $\begin{array}{c}0.2457 \\
0.3933\end{array}$ & $\begin{array}{c}7.6275 \\
0.3207\end{array}$ & 0.5616 & 1.1856 & $\begin{array}{l}- \\
455.6912 *\end{array}$ \\
\hline & Conditional & $\begin{array}{l}{[2.6014} \\
3.9989]\end{array}$ & $\begin{array}{l}{[0.9997} \\
1.9997]\end{array}$ & $\begin{array}{l}\overline{-} \\
0.7280 *\end{array}$ & 0.0104 & $\begin{array}{c}0.2071 \\
0.4852\end{array}$ & $\begin{array}{l}8.2721 * \\
0.3669\end{array}$ & 0.1768 & $1.0296 *$ & -452.1387 \\
\hline \multirow{3}{*}{200} & $\begin{array}{c}\text { Matlab } \\
\text { (mvnrnd) }\end{array}$ & $\begin{array}{l}{[2.5994} \\
3.9995]\end{array}$ & $\begin{array}{l}{[1.0003} \\
2.0008]\end{array}$ & $\begin{array}{c}- \\
0.7019\end{array}$ & 0.0013 & $\begin{array}{c}0.0201 \\
0.9549\end{array}$ & $\begin{array}{l}7.7267 \\
0.3145\end{array}$ & 0.9884* & $1.2324 *$ & -1332.4 \\
\hline & Decomposition & $\begin{array}{l}{[2.5992} \\
4.0014]\end{array}$ & $\begin{array}{l}{[0.9999} \\
2.0025]\end{array}$ & -0.7266 & 0.0012* & $\begin{array}{c}0.4234 \\
0.0069\end{array}$ & $\begin{array}{c}9.1161 \\
0.0243\end{array}$ & 0.4155 & 2.3244 & -1334.0 \\
\hline & Conditional & $\begin{array}{l}{[2.6001} \\
4.0002]\end{array}$ & $\begin{array}{l}{[1.0006} \\
1.9998]\end{array}$ & -0.6959 & $0.0012 *$ & $\begin{array}{c}0.0445 \\
0.8295\end{array}$ & $\begin{array}{l}\text { 8.0473* }^{*} \\
0.4666\end{array}$ & 0.8811 & 1.2480 & $-1334.2 *$ \\
\hline
\end{tabular}


Table (3-5): Number times of excellence for each method and the total ratio to excellence

\begin{tabular}{|c|c|c|c|}
\hline \multirow{2}{*}{ Sample size } & \multicolumn{2}{|c|}{ Methods } \\
\cline { 2 - 4 } & $\begin{array}{c}\text { Matlab } \\
\text { (mvnrnd) }\end{array}$ & Decomposition & Conditional \\
\hline 15 & 3 & 2 & 2 \\
\hline 50 & 3 & 1 & 3 \\
\hline 100 & 3 & 0.5 & 2.5 \\
\hline 200 & 4 & 4.5 & 10.5 \\
\hline Total of each method & 13 & $16 \%$ & $37 \%$ \\
\hline Ratio & $47 \%$ & & 3 \\
\hline
\end{tabular}

Table (3-6): The average of all parameters and criterion of each method

\begin{tabular}{|l|c|c|c|c|c|c|c|c|c|c|c|}
\hline Methods & mean1 & mean2 & var1 & var2 & roh & MSE & skw & kur & P_JB & time & AIC \\
\hline $\begin{array}{l}\text { Matlab } \\
\text { (mvnrnd) }\end{array}$ & 2.600 & 4.002 & $\mathbf{0 . 9 9 9}$ & 1.996 & $\begin{array}{c}- \\
0.714\end{array}$ & 0.809 & $\mathbf{0 . 5 4 4}$ & 7.263 & 0.729 & 1.073 & - \\
\hline Decomposition & 2.599 & 4.001 & $\mathbf{0 . 9 9 9}$ & 2.000 & $\begin{array}{c}- \\
\mathbf{0 . 7 3 6}\end{array}$ & $\mathbf{0 . 8 2 6}$ & $\mathbf{0 . 3 4 6}$ & $\mathbf{7 . 1 4 2}$ & $\mathbf{0 . 6 3 8}$ & 1.482 & - \\
471.901 \\
\hline Conditional & $\mathbf{2 . 6 0 3}$ & 4.000 & 1.000 & 1.998 & $\begin{array}{c}- \\
\mathbf{0 . 7 1 8}\end{array}$ & $\mathbf{0 . 8 3 4}$ & $\mathbf{0 . 2 5 1}$ & $\mathbf{6 . 1 1 8}$ & $\mathbf{0 . 6 3 8}$ & 1.037 & - \\
471.107 \\
\hline
\end{tabular}

Table (3-7): Number times of excellence for each method and the total ratio to excellence according to the average of each method

\begin{tabular}{|c|c|c|c|}
\hline Methods & $\begin{array}{c}\text { Matlab } \\
\text { (mvnrnd) }\end{array}$ & Decomposition & Conditional \\
\hline Total of each method & 6 & 1 & $\mathbf{4}$ \\
\hline Ratio & $55 \%$ & $\mathbf{9 \%}$ & $\mathbf{3 6 \%}$ \\
\hline
\end{tabular}




\section{6- Discussion of the Results} following

Form the above tables we can discuss the

result:

1- We can see in the tables (3-1), (3-2) and (3-3) that the valve of $\widehat{\boldsymbol{\mu}}$ and $\widehat{\sum}$ are approach to the default value, which $\mu=\left[\begin{array}{c}2.6 \\ 4\end{array}\right], \Sigma=$ $\left[\begin{array}{cc}1 & -1 \\ -1 & 2\end{array}\right]$ when the sample size are increasing from the smaller (15) to greater (200) in all three methods .

2- Note that the criterion MSE, AIC in all simulation results in the tables (3-1),(3-2) and (3-3) above are inversely proportional to the sample size, which mean, when sample size is increasing the criterion MSE, AIC are decreasing .but the values of criterion skewness (sk) are approach to zero when the sample size is increasing in each method, also the values of kurtosis criterion( $\mathrm{ku}$ ) are nearly from (8) in each method when the sample size start to be increasing, in addition, the values of the joint criterion (p_JB) in every method and in all cases of sample size are greater than (0.05) then we accept the hypothesis $\mathrm{H} \circ$, which $\mathrm{H}_{\circ}$ : the data is belong to MVN

If $\mathrm{p}$-value $<0.05$ the hypothesis $\mathrm{H}_{\circ}$ is reject. Either $\mathrm{p}$-value $>0.05$ the hypothesis $\mathrm{H} \circ$ is not reject.

3- The table (3-4) shown the comparison between the simulation result of these methods ,such that the best value to the criterion MSE,AIC when sample size is(15),(50) is matlab (mvnrnd) method, and when sample size increasing (100) the best value of MSE is in the same method above but AIC in decomposition method, also when sample size is more increasing (200) we can see the same value (0.0012)to criterion MSE in decomposition ,conditional method, respectively and criterion AIC is just in conditional method .
4- In same table (3-4) of comparison the best value of criterion skewness when sample size is (100) and (200) in matlab (mvnrnd) method, but in decomposition when sample size is (15) while in conditional method when sample size is (50).

5- In table (3-5) we make a comparison to know the number of times of excellence and total ratio of excellence $\left(\right.$ ratio $\left.=\frac{\text { sub number }}{\text { total number }} * 100\right)$ for each method and we get that the upper ratio to the matlab (mvnrnd) method (47\%), while the second method (decomposition) have got on $(37 \%)$, and so be the matlab (mvnrnd) method is the best method to generate observation of multivariate normal distribution.

6- After that we put table (3-6) to get the average of all the parameter and criterion of each method, this average got from accumulated the four values of parameter and criterion in addition to correlation in all case of sample size after that we divided it by four, for example to get the value (2.600) of mean 1 in the same table, we do that

Mean1=

$(2.5975+2.5996+2.5980+2.5994) / 4=2.600$,

And so on for all other values, in addition to the table (3-7) contain the comparison of number of the times of excellence and the ratio to table (3-6), and finally we deduced that the best method is matlab (mvnrnd)method because it get $(55 \%)$ as a better ratio . 


\section{7- Appendixes (Programs)}

clear all;num=1;

while num $<4$

num=input('number of program 1 cond. 2 Dec.

3 Matlab?');

switch num

case 1

$\%$ program 1 Generate observation of

Multivariate Normal by conditional with

Box_Muller Method

disp('Result of conditional method')

$\mathrm{n}=$ input('sample size?');\% $\mathrm{n}$ is sample size.

Rep=input('R of Rep.?');\% number of

replications

t0=cputime();rand('seed',n);

$\mathrm{s} \_\mathrm{m}=0 ; \mathrm{ssme}=[00] ; \mathrm{ssva}=[00] ; \mathrm{SS} \_\mathrm{MS}=0$;

$\mathrm{mu}=[2.6 ; 4] ;$ sigma $=[1-1 ;-12]$;

rho $=\operatorname{sigma}(1,2) /\left(\operatorname{sigma}(1,1)^{*} \operatorname{sigma}(2,2)\right)^{\wedge} 0.5$;

l=length $(\mathrm{mu})$;

for $\mathrm{r}=1$ :Rep

$\mathrm{U}=$ unifrnd $(0,1,2, \mathrm{n}) ; \mathrm{U} 1=\mathrm{U}(1,:) ; \mathrm{U} 2=\mathrm{U}(2,:) ; \mathrm{ph}=2$

$*(\mathrm{pi}) * \mathrm{U} 1 ; \mathrm{R}=(-2 * \log (\mathrm{U} 2)) .^{\wedge} 0.5$;

$\mathrm{z} 1=\mathrm{R} . * \cos (\mathrm{ph}) ; \mathrm{z} 2=\mathrm{R} . * \sin (\mathrm{ph})$;

$\mathrm{x} 1=\mathrm{mu}(1,1)+\mathrm{z} 1 * \operatorname{sigma}(1,1)^{\wedge} 0.5$;

$\mathrm{y} 2=\mathrm{rho}^{*} \mathrm{z} 1+\operatorname{sqrt}\left(1-(\mathrm{rho})^{\wedge} 2\right)^{*} \mathrm{z} 2$;

$\mathrm{x} 2=\mathrm{mu}(2,1)+\operatorname{sigma}(2,2)^{\wedge} 0.5^{*} \mathrm{y} 2$;

$\mathrm{x}=[\mathrm{x} 1 ; \mathrm{x} 2]^{\prime} ; \mathrm{me}=\operatorname{mean}(\mathrm{x})$;

ssme $=$ ssme + me $;$ ssva $=$ ssva $+\operatorname{var}(\mathrm{x})$;

SS_MS=SS_MS+(me'-mu)*(me'-mu $)^{\prime}$;

end

me $=$ ssme/Rep

var=ssva/Rep

$\mathrm{MSE}=$ det $\left(\mathrm{SS} \_\mathrm{MS}\right) /(\mathrm{n}-1) / \operatorname{Rep}$

$\%$ MSE criterion for model

$\mathrm{AIC}=\mathrm{n} * \log (\mathrm{MSE})+2 * 1$

$\%$ AIC criterion for model

$\mathrm{co}=\operatorname{corr}(\mathrm{x}) ; \mathrm{rho} \_\mathrm{h}=\mathrm{co}(1,2)$

$\mathrm{X}=\mathrm{x}$;

$[\mathrm{n}, \mathrm{p}]=\operatorname{size}(\mathrm{X}) ;$ alpha $=0.05$;

$\operatorname{difT}=[]$;

for $\mathrm{j}=1: \mathrm{p}$

$\operatorname{difT}=[\operatorname{difT},(X(:, j)-\operatorname{mean}(X(:, j)))] ;$

end;

$\mathrm{S}=\operatorname{cov}(\mathrm{X}) ; \quad \%$ Variance-covariance matrix

$\mathrm{D}=\operatorname{difT} * \operatorname{inv}(\mathrm{S}) * \operatorname{difT} ; \%$ Mahalanobis'

distances matrix

$\mathrm{b} 1 \mathrm{p}=\left(\operatorname{sum}\left(\operatorname{sum}\left(D .^{\wedge} 3\right)\right)\right) / \mathrm{n}^{\wedge} 2 ; \%$ Multivariate

skewness coefficient

b2p = trace(D.^2) / n; \% Multivariate kurtosis

coefficient

$\mathrm{v}=(\mathrm{p} *(\mathrm{p}+1) *(\mathrm{p}+2)) / 6$

$\%$ Degrees of freedom

$\mathrm{g} 1=(\mathrm{n} * \mathrm{~b} 1 \mathrm{p}) / 6$;

$\%$ Skewness test statistic

(approximates to a chi-square distribution)

$\mathrm{P} 1=1-\operatorname{chi} 2 \operatorname{cdf}(\mathrm{g} 1, \mathrm{v}) ; \%$ Significance value of

skewness $\mathrm{g} 2=(\mathrm{b} 2 \mathrm{p}-(\mathrm{p} *(\mathrm{p}+2))) / \ldots$

$(\operatorname{sqrt}((8 * \mathrm{p} *(\mathrm{p}+2)) / \mathrm{n})) ; \quad \%$ Kurtosis test statistic (approximates to a unit-normal distribution)

P2 = 1-normcdf $(\operatorname{abs}(g 2)) ; \%$ Significance value of

kurtosis

$\mathrm{sk}=\mathrm{b} 1 \mathrm{p}$

$\mathrm{ku}=\mathrm{b} 2 \mathrm{p}$

stats.Ps $=\mathrm{P} 1$

stats.Pk = P2

$\mathrm{ks}=$ skewness $(\mathrm{X}) ; \mathrm{ku}=\mathrm{kurtosis}(\mathrm{X})-3$;

kwen=ks*ks';kur=ku*ku';

$\mathrm{jb}=\mathrm{n} / 6^{*}(\mathrm{kwen}+\mathrm{kur} / 4)$

$p_{-} j b=1-\operatorname{chi} 2 c d f(j b, v)$

Mean_Ku=p* $(\mathrm{p}+2) *(\mathrm{p}+1+\mathrm{n}) / \mathrm{n}$

time $=$ cputime ()$-\mathrm{t} 0$

case 2

$\%$ program 2 Generate observation of Multivariate

Normal by decomposition with inverse Method

disp('Result of Dec. method')

n=input('sample size?');

Rep=input('R of Rep.?');

$\mathrm{t} 0=$ cputime ();rand('seed',n);

s_m $=0 ;$ ssme $=\left[\begin{array}{ll}0 & 0\end{array}\right] ; \mathrm{ssva}=\left[\begin{array}{ll}0 & 0\end{array}\right] ; \mathrm{SS} \_\mathrm{MS}=0$;

$\mathrm{mu}=[2.6 ; 4] ; \operatorname{sigma}=[1-1 ;-12] ; \mathrm{l}=\mathrm{length}(\mathrm{mu}) ; \mathrm{A}=\operatorname{sigma}$;

$\mathrm{T}=\operatorname{chol}(\mathrm{A})$;

for $\mathrm{r}=1: \operatorname{Rep}$

$\mathrm{u}=\operatorname{rand}(\mathrm{n}, 2) ; \mathrm{Z}=\operatorname{norminv}(\mathrm{u}, 0,1) ; \mathrm{Y}=\mathrm{Z} * \mathrm{~T}$;

for $\mathrm{i}=1: \mathrm{n}$

for $\mathrm{j}=1: 2$

$X(i, j)=m u(j)+Y(i, j)$;

end

end

me $=$ mean $(X)$;

ssme $=$ ssme + me $;$ ssva $=$ ssva $+\operatorname{var}(\mathrm{X})$;

$\mathrm{SS} \_\mathrm{MS}=\mathrm{SS} \_\mathrm{MS}+\left(\mathrm{me}^{\prime}-\mathrm{mu}\right) *\left(\mathrm{me} \mathrm{e}^{\prime}-\mathrm{mu}\right)^{\prime}$;

end

me $=$ ssme $/$ Rep

var $=$ ssva/Rep

$\mathrm{MSE}=\mathrm{abs}\left(\operatorname{det}\left(\mathrm{SS} \_\mathrm{MS}\right)\right) /(\mathrm{n}-1) / \mathrm{Rep}$

$\%$ MSE criterion for model

$\mathrm{AIC}=\mathrm{n} * \log (\mathrm{MSE})+2 * 1$

$\%$ AIC criterion for model

$\mathrm{co}=\operatorname{corr}(\mathrm{X}) ; \mathrm{rho} \_\mathrm{h}=\mathrm{co}(1,2)$

$[\mathrm{n}, \mathrm{p}]=\operatorname{size}(\mathrm{X}) ;$ alpha $=0.05$;

$\operatorname{difT}=[]$;

for $\mathrm{j}=1: \mathrm{p}$

$\operatorname{difT}=[\operatorname{difT},(X(:, j)-\operatorname{mean}(X(:, \mathrm{j})))] ;$

end;

$\mathrm{S}=\operatorname{cov}(\mathrm{X}) ; \quad \%$ Variance-covariance matrix

$\mathrm{D}=\operatorname{difT} * \operatorname{inv}(\mathrm{S}) * \operatorname{difT} ; \%$ Mahalanobis' distances matrix

$\mathrm{b} 1 \mathrm{p}=\left(\operatorname{sum}\left(\operatorname{sum}\left(D .^{\wedge} 3\right)\right)\right) / \mathrm{n}^{\wedge} 2 ; \quad \%$ Multivariate

skewness coefficient

b2p $=\operatorname{trace}\left(D .^{\wedge} 2\right) / n ; \%$ Multivariate kurtosis coefficient

$\mathrm{v}=(\mathrm{p} *(\mathrm{p}+1) *(\mathrm{p}+2)) / 6 ; \quad \%$ Degrees of freedom $\mathrm{g} 1=(\mathrm{n} * \mathrm{~b} 1 \mathrm{p}) / 6 ; \quad \%$ Skewness test statistic

(approximates to a chi-square distribution) 
$\mathrm{P} 1=1-\operatorname{chi} 2 \mathrm{cdf}(\mathrm{g} 1, \mathrm{v}) ; \%$ Significance value of skewness $\mathrm{g} 2=(\mathrm{b} 2 \mathrm{p}-(\mathrm{p} *(\mathrm{p}+2))) / \ldots$

$(\operatorname{sqrt}((8 * \mathrm{p} *(\mathrm{p}+2)) / \mathrm{n})) ; \quad \%$ Kurtosis test statistic (approximates to a unit-normal distribution)

$\mathrm{P} 2=1$-normcdf $(\operatorname{abs}(\mathrm{g} 2)) ; \%$ Significance value of kurtosis

stats.Ps $=\mathrm{P} 1$

stats.Pk $=\mathrm{P} 2$

$\mathrm{sk}=\mathrm{b} 1 \mathrm{p}$

$\mathrm{ku}=\mathrm{b} 2 \mathrm{p}$

$\mathrm{ks}=$ skewness $(\mathrm{X}) ; \mathrm{ku}=\mathrm{kurtosis}(\mathrm{X})-3$;

kwen=ks*ks';kur=ku*ku';

$\mathrm{jb}=\mathrm{n} / 6^{*}(\mathrm{kwen}+\mathrm{kur} / 4)$

p_jb $=1-\operatorname{chi} 2 \operatorname{cdf}(j b, v)$

time $=$ cputime ()$-\mathrm{t} 0$

case 3

$\%$ program 3 Generate observation of Multivariate

Normal by Matlab function

disp('Result of MATLAB method')

$\mathrm{n}=$ input('sample size $\mathrm{n}$ ?');

$\% \mathrm{n}$ is sample size.

Rep=input('R of Rep.=');

t $0=$ cputime ()$;$ randn('seed',n);

$\mathrm{s} \_\mathrm{m}=0 ; \mathrm{ssme}=[00] ; \mathrm{ssva}=[00] ; \mathrm{SS} \_\mathrm{MS}=0$;

$\mathrm{mu}=[2.6 ; 4] ;$ sigma $=[1-1 ;-12] ; 1=\operatorname{length}(\mathrm{mu})$;

for $r=1: \operatorname{Rep}$

$\mathrm{x}=\operatorname{mvnrnd}(\mathrm{mu}, \operatorname{sigma}, \mathrm{n}) ; \mathrm{me}=$ mean $(\mathrm{x})$;

ssme $=$ ssme + me; ssva $=$ ssva $+\operatorname{var}(x)$;

SS_MS=SS_MS+(me'-mu $) *(m e '-m u) '$;

$$
\text { end }
$$

me $=$ ssme/Rep

var=ssva/Rep

$\mathrm{MSE}=\operatorname{det}\left(\mathrm{SS} \_\mathrm{MS}\right) /(\mathrm{n}-1) / \mathrm{Rep}$

$\%$ MSE criterion for model

$\mathrm{AIC}=\mathrm{n} * \log (\mathrm{MSE})+2 * 1$

$\%$ AIC criterion for model

$\mathrm{co}=\operatorname{corr}(\mathrm{x}) ; \mathrm{rho} \_\mathrm{h}=\mathrm{co}(1,2)$

$\mathrm{X}=\mathrm{x}$;

$[\mathrm{n}, \mathrm{p}]=\operatorname{size}(\mathrm{X}) ;$ alpha $=0.05$;

$\operatorname{difT}=[]$;

for $\mathrm{j}=1: \mathrm{p}$

$\operatorname{difT}=[\operatorname{difT},(X(:, \mathrm{j})-\operatorname{mean}(\mathrm{X}(: \mathrm{j})))] ;$

end;

$\mathrm{S}=\operatorname{cov}(\mathrm{X}) ; \quad \%$ Variance-covariance matrix

$\mathrm{D}=\operatorname{difT} * \operatorname{inv}(\mathrm{S}) * \operatorname{difT} ; \%$ Mahalanobis' distances

matrix

$\mathrm{b} 1 \mathrm{p}=\left(\operatorname{sum}\left(\operatorname{sum}\left(D .^{\wedge} 3\right)\right)\right) / \mathrm{n}^{\wedge} 2 ; \%$ Multivariate

skewness coefficient

b2p = trace $\left(D .^{\wedge} 2\right) / n$; \% Multivariate kurtosis

coefficient

$\mathrm{v}=\left(\mathrm{p}^{*}(\mathrm{p}+1) *(\mathrm{p}+2)\right) / 6 ; \quad \%$ Degrees of freedom

$\mathrm{g} 1=(\mathrm{n} * \mathrm{~b} 1 \mathrm{p}) / 6 ; \quad \%$ Skewness test statistic

(approximates to a chi-square distribution)

$\mathrm{P} 1=1-\operatorname{chi} 2 \operatorname{cdf}(\mathrm{g} 1, \mathrm{v}) ; \%$ Significance value of

skewness

$\mathrm{g} 2=(\mathrm{b} 2 \mathrm{p}-(\mathrm{p} *(\mathrm{p}+2))) / \ldots$ $(\operatorname{sqrt}((8 * \mathrm{p} *(\mathrm{p}+2)) / \mathrm{n})) ; \quad \%$ Kurtosis test statistic (approximates to a unit-normal distribution)

P2 = 1-normcdf $(\operatorname{abs}(g 2)) ; \%$ Significance value of kurtosis

$\mathrm{sk}=\mathrm{b} 1 \mathrm{p}$

$\mathrm{ku}=\mathrm{b} 2 \mathrm{p}$

stats.Ps = P1

stats.Pk = P2

$\mathrm{ks}=$ skewness $(\mathrm{X}) ; \mathrm{ku}=\mathrm{kurtosis}(\mathrm{X})-3$;

kwen=ks*ks';kur=ku*ku';

$\mathrm{jb}=\mathrm{n} / 6^{*}(\mathrm{kwen}+\mathrm{kur} / 4)$

$p_{-} j b=1-\operatorname{chi} 2 c d f(j b, v)$

Mean_Ku=p* $(\mathrm{p}+2) *(\mathrm{p}+1+\mathrm{n}) / \mathrm{n}$

time $=$ cputime ()$-\mathrm{t} 0$

otherwise

disp('End of select')

break

end

end

\section{Reference}

[1] Anderson, T.W., An Introduction to multivariate statistical analysis, John Wiley and Sons, (1984).

[2] Rencher, Alvin C., Methods of multivariate analysis, Second edition, Brigham young university, p. 83, (2002).

[3] N.Balakrishnan, chin-diew lai, continuous Bivariate distributions, Second edition, 633,636, 2008.

[4] Bischoff, wolfgang, fieger, Werner, Characterization of the multivariate normal distribution by conditional normal distributions, University of Karlsruhe, p.240, (1991).

[5] Patrick J. Farrell, Matias Salibian-Barrera and Katarzyna Naczk, On test for multivariate normality

and associated simulation studies, Journal of statistical computation and simulation, Vol.77, No. 12, 1065-1080, (2007).

[6] Chun-Chao Wang, Amatlab package for multivariate normality test, Journal of statistical Computation and simulation, (2013).

[7] Hanusz, zofia and tarasinska,Joanna, On multivariate normality tests using skewness and kurtosis, colloquium biometricum 44 , 139-148, (2014).

zofia.hanusz@up.lublin.pl , Joanna.tarasinska@up.lublin.pl 
طرائق محاكاة للتوزيع الطبيعي متعدد المتغيرات

\author{
علي حسين بتور إسراء عبد الرضا باقر \\ كلية التربية للبنات \\ جامعة الكوفة
}

\author{
فاضل عبد العباس العابدي \\ الكلية التقتية الادارية العابة \\ جامعة الفرات الاوسط التقنية
}

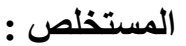

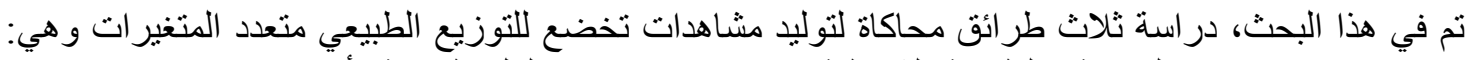

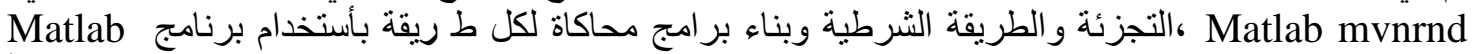

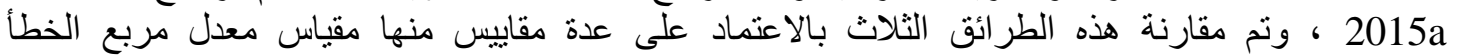
،مقياس اكاكي ومقياس الالتو اء و التفرطح kur, skw، على التو الي بالاضافة الى مقياس سرعة التنفيذ لكل 